\title{
Factor Structure of the Negative Symptom Assessment
}

\author{
Bradley N. Axelrod, Robert S. Goldman, John L. Woodard, and \\ Larry D. Alphs
}

Received June 8, 1993; revised version received September 28, 1993; accepted October 11, 1993.

\begin{abstract}
The factor structure of the Negative Symptom Assessment (NSA), a standardized negative symptoms rating scale, was systematically evaluated in a group of 223 inpatients with schizophrenia. Confirmatory factor analyses found that a six-factor model best described the NSA. More specifically, the domains of Communication, Emotion/Affect, Social Involvement, Motivation, Gross Cognition, and Retardation characterized the rating scale. This latent structure of the NSA is consistent with a multidimensional conceptualization of negative symptoms.
\end{abstract}

Key Words. Schizophrenia, factor analysis, symptomatology.

Positive symptoms, such as hallucinations and delusions, are traditionally recognized as hallmark features of schizophrenia. In recent years, the presence of another group of characteristics has also been increasingly recognized as defining clinical features of schizophrenia (Strauss et al., 1974; Crow, 1980). These "negative" symptoms include diminished affect, poverty of speech, avolition, and social withdrawal (Crow, 1980; Andreasen and Olsen, 1982). Over eight rating scales (Fenton and McGlashan, 1992) have been developed for the purpose of assessing negative symptoms in schizophrenia. Despite the proliferation of these scales, concurrent validity is poor (Fenton and McGlashan, 1992), and the construct validity of any one instrument remains questionable.

The Negative Symptom Assessment (NSA), a 26-item rating scale, was explicitly developed to characterize negative symptoms in a more standardized and comprehensive manner (Alphs et al., 1989). Specifically, the NSA assesses a potentially greater range of negative symptoms, includes better defined and anchored items, and is administered using a standardized structured interview. On the basis of criteria set forth by Cicchetti and Sparrow (1981) for interpreting intraclass correlation coefficients, interrater and test-retest reliabilities on the NSA fall in the excellent range (Alphs et al., 1989). Axelrod and Alphs (1993) found that individuals, previously unfamiliar with the NSA, were able to assess negative symptoms at a level consistent with an expert after a single 30 -minute training session.

Bradley N. Axelrod, Ph.D., and Robert S. Goldman, Ph.D., are Staff Psychologists, Department of Veterans Affairs Medical Center, Allen Park and Ann Arbor, MI, respectively. Dr. Goldman is also Assistant Professor, University of Michigan, Ann Arbor, MI. John L. Woodard, Ph.D., is Assistant Professor of Neurology, Emory University School of Medicine, Atlanta, GA. Larry D. Alphs, M.D., Ph.D., is Associate Director, Sandoz Research Institute, Central Nervous System Division, East Hanover, NJ. (Reprint requests to Dr. B.N. Axelrod, Psychology Service, 116B, DVA Medical Center, Soutfield \& Outer Dr., Allen Park, MI 48101, USA.)

0165-1781/94/\$07.00 인 1994 Elsevier Science Ireland Ltd. 
The NSA was created on the assumption that negative symptoms are multidimensional in nature. If it were shown that negative symptoms are clinically multidimensional, then it would be possible to evaluate differential medication efficacy, treatment outcome, and pathophysiological significance of individual negative symptom dimensions. Most existing scales typically make use of global scores and ignore the possibility that the broad pool of items may, in fact, measure several possible dimensions of negative symptoms. The NSA was developed to assess five distinct dimensions of negative symptoms (see Table 1). Specifically, the 26 NSA items were devised to tap the dimensions of impaired Communication, disturbed Affect/ Emotion, reduced Social Involvement, reduced Motivation, and impaired Gross Cognition. A factor validation was performed to assess the assumption that these five symptom clusters represent distinct dimensions in the clinical context. An exploratory principal components factor analysis of the NSA with 88 schizophrenic and 12 schizoaffective patients (Alphs et al., 1989) revealed that the 26 items were best characterized by six, not five, separate factors. As a result of the structure obtained from the above analysis, a revised six-factor theorctical model was established (see Table 1). The new empirically derived model differs primarily from the rationally developed model by the emergence of a Retardation factor.

The purpose of the present study was to perform an independent validation of the dimensional structure of the NSA. It would be inappropriate to conduct another exploratory factor analysis (EFA) since EFA is used to generate theoretical structures based on covariance patterns that are present in a data set and may therefore not be generalizable to other samples. In contrast, a confirmatory factor analysis (CFA) statistically tests the adequacy of specific theoretical models that are based on a priori constructs. CFA is the most appropriate method for evaluating the dimensional structure of a rating instrument. To this end, the current study contrasts the five- and six-factor models of the NSA to detcrminc which of the models best represents the latent structure of the rating scale as applied to schizophrenia.

\section{Methods}

Subjects. The study group comprised inpatients from 12 psychiatric settings (six Department of Veterans Affairs Medical Centers, six State Hospitals). All subjects met $D S M-I I /-R$ criteria for a diagnosis of schizophrenia (American Psychiatric Association, 1987) and were participants in an inpatient medication study. They averaged 40.0 years of age $(S D=11.0)$, and the mean age of onset of illness was 22.2 years $(S D=5.9)$. Subjects were administered the structured NSA interview approximately 7.0 days $(\mathrm{SD}=1.4)$ after hospitalization. Twentyseven clinical raters administered the structured interviews and achieved excellent consistency of ratings relative to an expert rater (for a discussion of the training, see Axelrod and Alphs, 1993). Of the subjects, 220 had been on placebo for 4-7 days at the time of assessment and three had been free of medication for an indeterminant length of time.

Procedure. A confirmatory factor analytic procedure (EQS; Bentler, 1989) was used to compare the two NSA models (Table 1) with the null model. A null model assumes no dimensional structure present in the scale and that each item therefore constitutes its own separate factor. In addition, a single factor model of the NSA was evaluated to test the possibility that negative symptoms are in fact unidimensional, not multidimensional.

A maximum likelihood CFA was performed for each of the models. Preliminary analyses revealed multivariate nonnormality of item ratings. A Robust analysis was performed, as it is 
less sensitive to violations of normality (cf. Bentler, 1992). Such an analysis results in the computation of the Satorra-Bentler $\chi^{2}$ test, which better approximates the $\chi^{2}$ distribution (Bentler, 1989).

Table 1. Item structure for models of the Negative Symptom Assessment

\begin{tabular}{lcc}
\hline Item & 5-factor model & 6-factor model \\
\hline Prolonged time to respond & 1 & 1 \\
Restricted speech quantity & 1 & 1 \\
Impoverished speech content & 1 & 1 \\
Failure to answer & 1 & 1 \\
Slow speech & 1 & 6 \\
Blocked speech & 1 & 1 \\
Monotonous speech & 1 & 6 \\
Muted speech & 1 & 6 \\
Inarticulate speech & 1 & 1 \\
Reduced expressive gestures & 1 & 6 \\
Blank, expressionless face & 2 & 2 \\
Emotion: reduced range & 2 & 2 \\
Affect: reduced modulation & 2 & 2 \\
Affect: reduced display & 2 & 2 \\
Reduced social drive & 3 & 3 \\
Poor rapport with interviewer & 3 & 3 \\
Avoids looking with interviewer & 3 & 3 \\
Reduced sexual interest & 3 & 3 \\
Poor grooming and hygiene & 4 & 4 \\
Reduced sense of purpose & 4 & 4 \\
Reduced hobbies and interests & 4 & 4 \\
Reduced daily activity & 4 & 4 \\
Poor abstraction & 5 & 5 \\
Poor memory & 5 & 5 \\
Temporal disorientation & 5 & 5 \\
Slowed movements & $\mathrm{x}$ & $\mathrm{x}$ \\
\hline
\end{tabular}

Note. $x=$ item not included in model. The numbers correspond to distinct factors: 1 -Communication. 2-Emotion/Affect. 3-Social Involvement. 4-Motivation. 5-Gross Cognition. 6-Retardation.

\section{Results}

Table 2 presents the results of the CFAs. Item 26 (Slowed Movements) of the NSA was removed from the analyses of the models due to its linear dependence on other items in the scale. The first row of Table 2 shows the model $X^{2}: d f$ ratio for each of the theoretical models. Bentler (1989) stated that the Akaike Information Criterion (AIC) seen in the second row of Table 2 takes into account the goodness of fit relative to the number of parameters. When multiple models are compared, the lower AIC represents a more useful model. In this study, the six-factor model clearly has the lowest AIC. In the third row, the Satorra-Bentler $\chi^{2}: d f$ ratio takes into account departures from normality in approximating the distribution (Bentler, 1989). The lower the ratios are relative to the ratio for the null model, the better the theoretical model approximates the data. As can be seen in Table 2, each of the 
Table 2. Goodness-of-fit indices for factor structure models of the Negative Symptom Assessment

\begin{tabular}{lcccc}
\hline Index & $\begin{array}{c}5- \\
\text { factor }\end{array}$ & $\begin{array}{c}6- \\
\text { factor }\end{array}$ & $\begin{array}{c}\text { Unitary } \\
\text { model }\end{array}$ & $\begin{array}{c}\text { Null } \\
\text { model }\end{array}$ \\
\hline$x^{2} \div d f$ & 2.9 & 2.5 & 4.5 & 11.2 \\
Akaike Information Criterion & 234 & 160 & 759 & 2940 \\
Satorra-Bentler $\chi^{2} \div d f$ & 2.5 & 2.2 & 3.9 & \\
Bentler-Bonnett normed fit index & 0.77 & 0.81 & 0.62 & \\
Non-normed fit index & 0.82 & 0.85 & 0.65 & \\
Comparative fit index & 0.84 & 0.87 & 0.68 & \\
\hline
\end{tabular}

models represents a substantial improvement over the null model. The BentlerBonnett, non-normed, and comparative fit indices are ratios that index the degree of deviation for each model relative to the null model. A high fit index indicates that the tested model better approximates the structure of the scale than does the null model. Fit indices $>0.9$ are desirable (Bentler, 1989).

Comparison of the models indicates that the multidimensional models were clearly superior to the single factor model. Evidence for this assertion is seen in the lower fit indices, higher $\chi^{2}: d f$ ratios, and overall Satorra-Bentler $\chi^{2}$ analyses. Nested models can be compared with each other by examining the $\chi^{2}$ differences. Direct statistical comparisons revealed that the one-factor model was significantly inferior to both the five-factor $\left(x^{2}=512, d f=34, p<0.001\right)$ and six-factor $\left(x^{2}=600, d f=39, p<0.001\right)$ models. With regard to the multidimensional models, the six-factor model represents a significant improvement in fit over the original five-factor theoretical model of the NSA $\left(x^{2}=88, d f=5, p<0.001\right)$.

In a closer examination of the six-factor model, inspection of the standardized residuals (i.e., correlations not adequately accounted for by the model) for the items revealed the residuals were symmetrical and that all were lower than 0.3 . This finding suggests that the six-factor model satisfactorily accounted for all of its items. Table 3 shows the factor loadings for the maximum likelihood solution. The $z$-score estimates for each item and their related factor loading were significantly different from zero $(p<0.0001)$, providing additional support for the latent structure of the NSA. Table 4 shows the intercorrelation matrix for the factors from the six-factor model. As can be seen in Table 4, all of the factors are significantly interrelated. This finding suggests that while the NSA is a multidimensional instrument with respect to measuring a number of aspects of negative symptoms, the dimensions themselves are not orthogonal to each other.

\section{Discussion}

The present study supports the sensitivity of the NSA in tapping multiple facets of negative symptoms in schizophrenia. We found that a theoretically derived and empirically revised six-factor model was the best of the models tested in characterizing the latent structure of this rating scale. Specifically, the NSA measures Communication, Retardation, Emotion/Affect, Social Involvement, Motivation, and Gross Cognition. Except for the addition of the Retardation factor, 
Table 3. Factor loadings for the six-factor model

\begin{tabular}{|c|c|c|c|c|c|c|}
\hline Item & 1 & II & III & IV & V & VI \\
\hline Prolonged time to respond & 0.82 & 0 & 0 & 0 & 0 & 0 \\
\hline Restricted speech quantity & 0.83 & 0 & 0 & 0 & 0 & 0 \\
\hline Impoverished speech content & 0.59 & 0 & 0 & 0 & 0 & 0 \\
\hline Failure to answer & 0.70 & 0 & 0 & 0 & 0 & 0 \\
\hline Slow speech & 0 & 0 & 0 & 0 & 0 & 0.72 \\
\hline Blocked speech & 0.57 & 0 & 0 & 0 & 0 & 0 \\
\hline Monotonous speech & 0 & 0 & 0 & 0 & 0 & 0.80 \\
\hline Muted speech & 0 & 0 & 0 & 0 & 0 & 0.57 \\
\hline Inarticulate speech & 0.37 & 0 & 0 & 0 & 0 & 0 \\
\hline Reduced expressive gestures & 0 & 0 & 0 & 0 & 0 & 0.82 \\
\hline Blank, expressionless face & 0 & 0.85 & 0 & 0 & 0 & 0 \\
\hline Emotion: reduced range & 0 & 0.77 & 0 & 0 & 0 & 0 \\
\hline Affect: reduced modulation & 0 & 0.88 & 0 & 0 & 0 & 0 \\
\hline Affect: reduced display & 0 & 0.73 & 0 & 0 & 0 & 0 \\
\hline Reduced social drive & 0 & 0 & 0.77 & 0 & 0 & 0 \\
\hline Poor rapport with interviewer & 0 & 0 & 0.80 & 0 & 0 & 0 \\
\hline Avoids looking at interviewer & 0 & 0 & 0.61 & 0 & 0 & 0 \\
\hline Reduced sexual interest & 0 & 0 & 0.43 & 0 & 0 & 0 \\
\hline Poor grooming and hygiene & 0 & 0 & 0 & 0.40 & 0 & 0 \\
\hline Reduced sense of purpose & 0 & 0 & 0 & 0.83 & 0 & 0 \\
\hline Reduced hobbies and interests & 0 & 0 & 0 & 0.94 & 0 & 0 \\
\hline Reduced daily activity & 0 & 0 & 0 & 0.87 & 0 & 0 \\
\hline Poor abstraction & 0 & 0 & 0 & 0 & 0.64 & 0 \\
\hline Poor memory & 0 & 0 & 0 & 0 & 0.68 & 0 \\
\hline Temporal disorientation & 0 & 0 & 0 & 0 & 0.59 & 0 \\
\hline
\end{tabular}

Note. I-Communication. II-Emotion/Affect. III-Social Involvement. IV-Motivation. V-Gross Cognition. VI-Retardation.

this model is generally consistent with the initial structure of this scale (Alphs et al., 1989). This basic model is also comparable to current notions of what phenomenologically constitutes negative symptoms (Andreasen and Olsen, 1982; Tandon and Greden, 1991). That is, most rating instruments of negative symptoms include items related to poor social functioning, impaired communication, flattened affect, impaired cognition, and poor motivation (Fenton and McGlashan, 1992).

Raskin et al. (1993) reported the NSA to be unidimensional based on high internal consistency in the context of performing an exploratory factor analysis. Results from the present population also revealed high internal consistency (coefficient $\alpha=0.94$ ), suggesting that the NSA items may homogeneously sample from the domain of negative symptoms. While the items homogeneously sample this domain, the current CFA suggests that there is a meaningful multidimensionality to the domain of negative symptoms. The high intercorrelations of the NSA factors imply that the multiple dimensions are not completely distinct.

The fact that an instrument such as the NSA can have high internal consistency and also be multidimensional may be somewhat unclear. A measure can assess one trait (i.e., be internally consistent) yet contain several dimensions of that trait. For 
Table 4. Intercorrelations of the factors in the six-factor model

\begin{tabular}{lcccccc}
\hline Factor & I & II & III & IV & V & VI \\
\hline I. Communication & - & 0.64 & 0.80 & 0.52 & 0.52 & 0.78 \\
II. Emotion/Affect & - & - & 0.78 & 0.56 & 0.50 & 0.89 \\
II. Social Involvement & - & - & - & 0.78 & 0.61 & 0.83 \\
IV. Motivation & - & - & - & - & 0.49 & 0.55 \\
V. Gross Cognition & - & - & - & - & - & 0.37 \\
VI. Retardation & - & - & - & - & - & - \\
\hline
\end{tabular}

example, depressive symptoms include vegetative, cognitive, affective, and social dimensions. The unitary clinical construct of depression is therefore multidimensional. Similarly, the results of the present study would suggest that the NSA is a multifactorial measure of the unitary construct of negative symptoms.

While negative symptoms have been extensively and consistently described in the literature beginning with Bleuler (1911/1950), no empirical "gold standard" exists for their measurement. A number of theoretically driven negative symptom rating scales have proliferated in the literature since 1982 (Andreasen and Olsen), but there is little agreement about the meaningfulness of the scores from these measures. While total scores are highly reliable and the instruments are intercorrelated, the syndrome definition of negative symptoms varies across instruments (Fenton and McGlashan, 1992). There clearly is a need to establish whether negative symptoms are in fact multidimensional and to determine the ability of different negative symptom scales to evaluate the dimensions. Empirical validation studies, such as the present one, may scrve as a departure point from which a multidimensional definition of a negative symptom syndrome could be derived. The multidimensional constructs would then need to be systematically examined using different scales purportedly measuring the same negative symptom constructs (i.e., uni-trait/multi-method; Campbell and Fiske, 1959).

Acknowledgment. This project was completed with the support of Merck, Sharp \& Dohme.

\section{References}

Alphs, L.D.; Summerfelt, A.; Lann, H.; and Muller, R.J. The Negative Symptom Assessment: A new instrument to assess negative symptoms in schizophrenia. Psychopharmacology Bulletin, 25:159-163, 1989

American Psychiatric Association. DSM-III-R: Diagnostic and Statistical Manual of Mental Disorders. 3rd ed., revised. Washington, DC: American Psychiatric Press, 1987.

Andreasen, N.C., and Olsen, S. Negative vs. positive schizophrenia: Definition and validation. Archives of General Psychiatry, 39:789-794, 1982.

Axelrod, B.N., and Alphs, L.D. Training novice raters on the Negative Symptom Assessment Scale. Schizophrenia Research, 9:25-28, 1993.

Bentler, P.M. EQS: Structural Equations Program Manual. Los Angeles: BMDP Statistical Software, Inc., 1989. 
Bentler, P.M. On the fit of models to covariances and methodology to the "Bulletin." Psychological Bulletin, 112:400-404, 1992.

Bleuler, E. Dementia Praecox or the Group of Schizophrenias. (1911) New York: International Universities Press, 1950.

Campbell, D.T., and Fiske, D.W. Convergent and discriminant validation by the multitraitmultimethod matrix. Psychological Bulletin, 59:81-105, 1959.

Cicchetti, D.V., and Sparrow, S.S. Developing criteria for establishing interrater reliability of specific items: Applications to assessment of adaptive behavior. American Journal of Mental Deficiency, 86:127-137, 1981.

Crow, T.J. Molecular pathology of schizophrenia: More than one disease process? British Journal of Medicine, 280:66-68, 1980.

Fenton, W.S., and McGlashan, T.H. Testing systems for assessment of negative symptoms in schizophrenia. Archives of General Psychiatry, 49:179-184, 1992.

Raskin, A.; Pelchat, R.; Sood, R.; Alphs, L.D.; and Levine, J. Negative Symptom Assessment of chronic schizophrenics. Schizophrenia Bulletin, 19:627-635, 1993.

Strauss, J.S.; Carpenter, W.T.; and Bartko, J.J. An approach to the diagnosis and understanding of schizophrenia: Speculations on the processes that underlie schizophrenic symptoms and signs. Schizophrenia Bulletin, 1 (Experimental Issue No. 11):61-69, 1974.

Tandon, R., and Greden, J.F. Negative symptoms of schizophrenia: The need for conceptual clarity. Biological Psychiatry, 30:321-325, 1991. 